PACS 32.15RM;

УДК 539.184

\title{
SENSING THE ELECTRIC AND MAGNETIC MOMENTS \\ OF A NUCLEUS IN THE N-LIKE ION OF ${ }_{83}^{209} \mathrm{Bi}$ (За матеріалами доповіді на конференції СЕМСТ-2)
}

\author{
O. Yu. Khetselius, E. P. Gurnitskaya
}

Odessa National Polytechnical University, Odessa

\begin{abstract}
\author{
SENSING THE ELECTRIC AND MAGNETIC MOMENTS \\ OF A NUCLEUS IN THE N-LIKE ION OF ${ }_{83}^{209} \mathrm{Bi}$
}

O. Yu. Khetselius, E. P. Gurnitskaya
\end{abstract}

It has been carried out sensing and estimating the magnetic and electric moments of nucleus in the N-like ${ }_{83}^{203} \mathrm{Bi}^{76+}$ ion on the basis of gauge-invariant QED perturbation theory calculation with an account of correlation, nuclear and QED effects.

Key words: estimate, nuclear electric and magnetic moments, Bi

\section{Анотація \\ ДЕТЕКТУВАННЯ ЕЛЕКТРИЧНОГО І МАГНІТНОГО МОМЕНТІВ ЯДРА В N-ПОДІБНОМУ ІОНІ ${ }_{83}^{209} \mathrm{Bi}$ \\ О. Ю. Хещеліус, О. П. Гурницька}

Виконано детектування та оцінку електричного та магнітного моментів ядра у $\mathrm{N}$-подібному ${ }_{83}^{203} \mathrm{Bi}^{76+}$ іоні на підставі калібровочно-інваріантної КЕД теорії збурень з урахуванням кореляційних, ядерних та КЕД ефектів.

Ключові слова: оцінка, ядерний електричний і магнітний моменти, вісмут

\section{Аннотация}

\section{ДЕТЕКТРОВАНИЕ ЭЛЕКТРИЧЕСКОГО И МАГНИТНОГО МОМЕНТОВ ЯДРА В N-ПОДОБНОМ ИОНЕ ${ }_{83}^{209} \mathrm{Bi}$}

\section{О. Ю. Хещелиус, Е. П. Гурницкая}

Выполнено детектирование и оценка электрического и магнитного моментов ядра в Nподобном ${ }_{83}^{203} \mathrm{Bi}^{76+}$ ионе на основе калибровочно-инвариантной КЭД теории возмущений с учетом корреляционных, ядерных и КЭД эффектов.

Ключевые слова: оценка, ядерный электрический и магнитный моменты, висмут

In last years a sensing the hyperfine structure (HFS) parameters and nuclear quadrupole moments for different heavy elements attracts a great interest (e.g.[1-16]). It is provided by necessity of further developing the modern as atomic and as nuclear theories. From the other side, a great progress in experiments has been achieved [1-4]. Recent accurate measurements of the HFS of highly charged 
ions and in particular, of the HFS for the ground state of the ${ }_{83}^{203} \mathrm{Bi}^{82+}$ [4] not only provide the possibility for testing the quantum electrodynamics (QED) in strong fields, but also sensing the electric charge and magnetic moment distributions inside the nucleus [1-11]. Theoretical calculations fulfilled during the last several years apart from the basis Fermi-Breit relativistic contributions also include the magnetic dipole moment distribution inside the nucleus (Bohr-Weisskopf effect) and radiative QED corrections (e.g. [1-5,13,18,19,21]). These corrections are calculated in the "external field" approximation, where the nucleus is considered as a source of an external filed for the electron.[15]. In calculations of the heavy ions the well known multi-configuration (MC) Dirac-Fock (DF) approach is widely used (e.g. $[1,2,5])$. It provides the most reliable version of calculation for atomic systems. More effective method, based on the QED perturbation theory (PT), has been developed in the series of papers [7-22]. The further improvement of this method is connected with using the gauge invariant procedures of generating relativistic orbitals basis's and more correct treating the nuclear and radiative QED effects. In paper by Labzowsky et al [5] the dynamical proton model of the HFS of ground state in the ${ }_{83}^{203} \mathrm{Bi}^{82+}$ is used. It takes into account explicitly the motion of the outer proton inside the ${ }_{83}^{203} \mathrm{Bi}^{82+}$ nucleus by means of the WoodSaxon potential. The starting point for evaluating the interelectron interaction corrections is the local DF version. Two advantages of the model should be noted: i). using standard QED rules for calculating radiative corrections; ii). automatical account for magnetic moment and electric charge distribution inside the nucleus.

From experimental point of view the $\mathrm{H}$-like ion ${ }_{83}^{203} \mathrm{Bi}^{82+}$ is not the ideal candidate for the measurement the HFS parameters since the lifetime broadening of the excited state $2 \mathrm{p}_{3 / 2}(\sim 100 \mathrm{eV})$ is apparently much larger than the HFS splitting $\left(\sim 5 \cdot 10^{-2} \mathrm{eV}\right)$ [5]. The situation looks more attractable for the excited $1 \mathrm{~s}^{2} 2 \mathrm{p}_{3 / 2}$ state of the Li-like ${ }_{83}^{203} \mathrm{Bi}^{82+}$ ion, since here the trasnsition energy to the ground $1 \mathrm{~s}^{2} 2 \mathrm{~s}_{1 / 2}$ state is more than one order of magnitude smaller and therefore the transition probability (width) is of the order of $0,1 \mathrm{eV}$. Moe favourable situation occurs gfor boron-like and nitrogen-like ${ }_{83}^{203} \mathrm{Bi}^{82+}$ ions. In the last case the $2 p_{3 / 2}$ state should be the ground state. Regarding value of the electric quadrupole moment $Q$ for the ${ }_{83}^{203} \mathrm{Bi}^{82+}$ nucleus one should mention that a new independent $Q\left({ }_{83}^{203} \mathrm{Bi}^{82+}\right)$ definition from experiments with highly charged ions would still desirable [5]. An accurate determination of $Q($ ${ }_{83}^{203} \mathrm{Bi}^{82+}$ ) from the HFS studying for neutral ${ }_{83}^{203} \mathrm{Bi}$ of $-0,516(15)\left(10^{-28} \mathrm{~m}^{2}\right)$ has been adopted as te new '2001' standard value, but it should be confirmed. The previous values ranged from $-0,370(26)$ to $-0,77(1)\left(10^{-28} \mathrm{~m}^{2}\right)$ (e.g. [4,5]). Of them the pionic value of $-0,516(15)\left(10^{-28} \mathrm{~m}^{2}\right)$ was choosen for the previous "1992' set of moments [4].

In this paper we carried out sensing and estimating the nuclear electric and magnetic moments of a nucleus in the $\mathrm{N}$-like ion of ${ }_{83}^{203} \mathrm{Bi}$ on the basis of gauge-invariant QED perturbation theory with an account of correlation (interelectron interaction corrections), nuclear and QED effects. In refs. [8$10,18-22]$ it has been developed a new ab initio approach to calculating spectra of heavy systems with account of relativistic, correlation effects, based on the QED qauge-invariant perturbation theory and new effective procedures for accounting the nuclear and radiative corrections in the hyperfine structure calculation.

Let us describe in brief the important moments of the calculation procedure.. Full details of the whole method and corresponding numerical procedure of calculation of the different characteristics, including the HFS constants and nuclear moments, can be found in $[8-10,18,19]$. The wave electron functions zeroth basis is found from the Dirac equation solution with potential, which includes the core $a b$ initio potential, electric, polarization potentials of nucleus (the gaussian form for charge distribution in the nucleus is used). All correlation corrections of the second and high orders of perturbation theory (electrons screening, particle-hole interaction etc.) are accounted for [6,7]. We set the charge distribution in the nucleus by the Gaussian function:

$$
\begin{gathered}
\rho(r \mid R)=\left(4 \gamma^{3 / 2} / \sqrt{\pi}\right) \exp \left(-\gamma r^{2}\right) \\
\int_{0}^{\infty} d r r^{2} \rho(r \mid R)=1 ; \int_{0}^{\infty} d r r^{3} \rho(r \mid R)=R
\end{gathered}
$$

Here $\gamma=4 / \pi R^{2}$; $\mathrm{R}$ is an effective nucleus radius, for which the standard Z-dependence is accepted [18]. Such definition of an effective nuclear radius is to be suitable at least as some zeroth approximation. Our approach allows to calculate the derivatives on $\mathrm{R}$ for characteristics which describe interaction of a nucleus with the external electrons. Then it is possible to make the redistribution of results when a radius $R$ is varied within the physically 
reasonable limits. As it has been shown in many papers (e.g. papers $[1-5,7,11]$ and refs there), the models with the Fermi and Gauss charge distribution in a nucleus are most widespread and more correct in comparison with the model of homogeneous ball charge distribution. For example, let us mention that a difference in values of the spectra levels energies is about several $\mathrm{cm}^{-1}[18,19]$. At the same time the most advanced model must be based on the direct solving of the corresponding nuclear task.. As example, one could mention different versions of the shell model with the Woods-Saxontype and spin-orbit potentials (e.g. refs.[20,21]). We have used the model [20]. The proton wavefunctions employed in the numerical calculation are the solutions of the Dirac equation with the potential [20] as follows:

$$
V-25 \cdot f(l, j) \cdot V^{\prime} / r
$$

with potential $V$ as:

$$
V_{0}-a\left[r^{4} / 4-r^{3}\left(R_{1}+R_{2}\right) / 3+r^{2} R_{1} R_{2} / 2\right], \ldots . . r<R_{2}
$$

0 , $r>R_{2}$

The advantages of the chosen potential in comparison with the well known Woods-Saxon potential given in [20]. Parameters are defined from the fitting condition for calculated and theoretical energies of the ground and first excited states (see [20]).

Let us suppose that the point-like nucleus posseses by some central potential $W(R)$. The transition to potential of the finite nucleus is realized by substituition $W(r)$ on

$$
\begin{gathered}
W(r \mid R)=W(r) \int_{0}^{r} d r r^{2} \rho(r \mid R)^{+} \\
+\int_{r}^{\infty} d r r^{2} W(r) \rho(r \mid R) .
\end{gathered}
$$

In our case the Coulomb potential for spherically symmetric density $\rho(r \mid R)$ is:

$$
V_{\text {nuсl }}(r \mid R)=-\left((1 / r) \int_{0}^{r} d r^{\prime} r^{2} \rho\left(r^{\prime} \mid R\right)^{+} \int_{r}^{\infty} d r^{\prime} r^{\prime} \rho\left(r^{\prime} \mid R\right)\right.
$$

This potential is calculated from solving the following system of differential equations:

$$
V^{\prime} \text { nucl }(r, R)=\left(1 / r^{2}\right) \int_{0}^{r} d r^{\prime} r^{\prime 2} \rho\left(r^{\prime}, R\right) \equiv\left(1 / r^{2}\right) y(r, R)
$$

$$
\begin{gathered}
y^{\prime}(r, R)=r^{2} \rho(r, R) \\
\rho^{\prime}(r, R)=-8 \gamma^{5 / 2} r / \sqrt{\pi} \exp \left(-\gamma r^{2}\right)= \\
=-2 \gamma r \rho(r, R)=-\frac{8 r}{\pi r^{2}} \rho(r, R)
\end{gathered}
$$

with the corresponding boundary conditions. Further one can write the Dirac-Fock -like equations for a multi-electron system \{core- $n l j\}$. Formally they fall into one-electron Dirac equations for the orbitals $n l j$ :

$$
\begin{aligned}
& \frac{\partial F}{\partial r}+(1+\chi) \frac{F}{r}-(\varepsilon+m-V) G=0 \\
& \frac{\partial G}{\partial r}+(1-\chi) \frac{G}{r}+(\varepsilon-m-V) F=0
\end{aligned}
$$

with large and small components $\mathrm{F}, \mathrm{G}$ and potential: $V(r)=2 V(r \mid$ core $)+V(r \mid n l j)+V_{e x}+V(r \mid R)$.

and $\chi$ is the Dirac quantum number. The potential $V(r)$ includes the electrical and polarization potentials of the nucleus. The part $V_{e x}$ accounts for exchange inter-electron interaction. The exchange effects are accounted for in the first two PT orders by the total inter-electron interaction [6,7]. The core electron density is defined by iteration algorithm within gauge invariant QED procedure [12]. The radiative QED (the self-energy part of the Lamb shift and the vacuum polarization contribtuion) are accounted for within the QED formalism $[8,18]$. The interelectron interaction corrections contribution ( derivative terms indicated by primes and arising due to the energy dependence of the effective interelectron potentials on energies [5]) is defined by experession:

$$
\begin{aligned}
& \Delta E= \sum_{\substack{a i \\
i \neq v}}\left\{\frac{V_{v i}\left[G_{i a v a}(0)-G_{i a a v}(\delta E)-\delta U_{i v}\right]}{E_{v}-E_{i}}+\right. \\
&+\left.\frac{\left[G_{v a i a}(0)-G_{v a a i}(\delta E)-\delta U_{v i}\right] V_{i v}}{E_{v}-E_{i}}\right\}+ \\
&+\sum_{\substack{i a \\
i \neq a}}\left\{\frac{V_{a i}\left[G_{i v a v}(0)-G_{i v v a}(-\delta E)\right]}{E_{a}-E_{i}}+\right. \\
&\left.+\frac{\left[G_{a v i v}(0)-G_{a v v i}(-\delta E)\right] \delta V_{i a}}{E_{v}-E_{i}}\right\}- \\
&-\sum_{w a} V_{v w} \frac{d G}{d E_{w a a v}}(\delta E)-\sum_{a b} V_{a b} \frac{d G}{d E_{b v v a}}(-\delta E)
\end{aligned}
$$


where

$$
\begin{gathered}
\delta \mathrm{E}=\mathrm{E}_{\mathrm{v}}-\mathrm{E}_{\mathrm{a}}, \\
G_{i j k l}(E)=\alpha^{2} \int d^{3} x_{1} \int d^{3} x_{2} \frac{\exp \left(i \sqrt{E^{2}+i \delta}\left|\vec{x}_{1}-\vec{x}_{2}\right|\right)}{\left|\vec{x}_{1}-\vec{x}_{2}\right|} . \\
\tilde{E}_{i}\left(x_{1}\right) \gamma_{\mu} \ddot{E}_{k}\left(x_{1}\right) \times \bar{E}_{j}\left(x_{2}\right) \gamma_{\mu} \ddot{E}_{l}\left(x_{2}\right)
\end{gathered}
$$

The matrix elements $V_{i j}$ are defined by

$$
V_{i j}=<i p\left|\frac{\vec{\alpha}_{p} \vec{\alpha}_{e}}{r_{e p}}\right| j p>
$$

or

$$
V_{i j}=<i p\left|\frac{1}{r_{e p}}\right| j p>
$$

where $\mid p>$ is the proton wavefunction. The symbol $v$ refers to the $2 p_{3 / 2}$ valence state. The summation over $i$ is extended over the entire Dirac spectrum and the indices $a, b$ run over the core electrons. The sum on $w$ runs over magnetic substates of the state $2 p_{3 / 2}$. The value $E_{\mathrm{i}}$ denotes the one-electron Dirac energies. The magnetic dipole and octupole interactions correspond to the first and the third terms of the partialwave expension for the first matrix element while the electric quadrupole interaction corresponds to the second term of the partial-wave expansion for the second matrix element. As for matrix elements $\delta U_{i j}$ they are defined as: $\left.\delta U_{i j}=<i|\delta U| j\right\rangle$, where $\delta U$ is the difference between the pure Coulomb potential and the arbitrary starting potential. In ref. [5] two options have been used: pure Coulomb potential of the nucleus (noninteracting electrons) and the local approximation to the DF potential. We have used the pure Coulomb potential and ab initio effective potential by Ivanov-Ivanova [6,7]. The HFS constants are defined by the radial integrals (c.f. $[8,18])$ :

$$
\begin{aligned}
\mathrm{A}= & \left\{\left[(4,32587) 10^{-4} \mathrm{Z}^{2} \chi \mathrm{g}_{\mathrm{I}}\right] /\left(4 \chi^{2}-1\right)\right\} \times \\
& \times \int_{0}^{\infty} d r r^{2} F(r) G(r) U\left(1 / r^{2}, R\right), \\
\mathrm{B}= & \left\{7.287810^{-7} \mathrm{Z}^{3} \mathrm{Q} /\left[\left(4 \chi^{2}-1\right) \mathrm{I}(\mathrm{I}-1)\right\} \times\right. \\
\times & \int_{0}^{\infty} d r r^{2}\left[F^{2}(r)+G^{2}(r) U\left(1 / r^{2}, R\right),\right.
\end{aligned}
$$

Here I is a spin of nucleus, $g_{I}$ is the Lande factor, $Q$ is a quadruple momentum of nucleus; radial integrals are calculated in the Coulomb units $(=3,57$ $10^{20} Z^{2} \mathrm{~m}^{-2} ;=6,17410^{30} Z^{3} \mathrm{~m}^{-3}$ ). Radial parts $F$ and $G$ of two components of the Dirac function for electron, which moves in the potential $V(r, R)+U(r, R)$, are defined by solution of the Dirac equations (perturbation theory zeroth order). The electric quadrupole spectroscopic HFS constant $B$ of an atomic state related to the electric field gradient $q$ and to the electric quadrupole moment $e Q$ of the nucleus in the following way: $B=e q Q / h$. So, in order to obtain the corresponding value of $Q$ it is necessary to combine the HFS constants data with the electric field gradient obtained from the QED perturbation theory formalism calculations in our approach.

We carried out the calculation of the nuclear electric and magnetic moments of the of ${ }_{83}^{209} \mathrm{Bi}$ nucleus in the $\mathrm{N}$-like ion. In table 1 we present the results for magnetic dipole moment $\mu$, electric quadrupole moment $Q$ and octupole magnetic moment $P$ together with data, obtained in the QED DF approximation and available experimental results $[4,5]$.

Table 1

The magnetic dipole moment $\mu$, the electric quadrupole moment $Q$ and the octupole magnetic moment $P$ for the ${ }_{83}^{209} \mathrm{Bi}$ nucleus

\begin{tabular}{|c|c|c|c|}
\hline Moments & Experiment & $\begin{array}{c}\text { Theory [5]: } \\
\text { DF }\end{array}$ & Present \\
\hline$\mu / \mu_{N}$ & $4,1106(2)$ & 3,98348 & 4,07137 \\
$Q\left(10^{-24} \mathrm{~cm}^{2}\right)$ & $-0,516(15)$ & $-0,27748$ & $-0,40382$ \\
$P /{ }_{N}\left(10^{-24} \mathrm{~cm}^{2}\right)$ & - & 5,36963 & 5,47013 \\
\hline
\end{tabular}

Let us remind that the key quantitative factor of agreement between theory and experiment is connected with the correct accounting for the interelectron correlations, finite size niclear, Breit and QED radiative corrections [1-5,8,18-22]. The well-known MCDF [1,2] method is not gauge-invariant one and an accounting of multi-particle interelectron correlations is not fully fulfilled, though in ref. [5] it has been used the gauge-invariant local DF version. From the other side, the contribution of the nuclear core-polarization effects caused by the valence proton and also the high order QED corrections can correspond the difference between theory and experiment for the electric quadrupole moment. In a case of the magnetic moments this inaccuracy is essentially compensated by another one namely the neglect of the anomalous magnetic moment within nuclear dynamical proton model.In conclusion let us underline that we have carried out sensing and estimating the nuclear magnetic and electric moments of the ${ }_{83}^{209} \mathrm{Bi}$ within the gauge-invariant QED PT with an account of the relativistic and correlation effects and reached sufficiently high accuracy. 


\section{Acknowledgement}

Authors would like to thank very much Prof. A.Glushkov for support and useful critical comments.

\section{References}

1. Bieron J., Pyykkő P., Degree of accuracy in determining the nuclear electric quadrupole moment of radium//Phys.Rev. A. - 2005. - Vol.71 - - P.0325021-8.

2. Bieron J., Pyykkő P., Jonsson P., Nuclear quadrupole moment of ${ }^{201} \mathrm{Hg} / /$ Phys. Rev. A. - 2005. - Vol.71. P.012502-1-8.

3. Schweppe J., Belkacem A., Blumenfeld L., Clayton N., Feinberg B., Gould H., Kostroum V.E., Levy L., Misawa S., Mowst J.R. and Priuor M.H. Measurement of the Lamb shift in lithiumlike uranium $\left(\mathrm{U}^{89+}\right) / /$ Phys.Rev.Lett. - 1998-Vol.66. P.1434-1437..

4. Klaft I.,Borneis S., Engel T., Fricke B., Grieser R., Huber G., Kuhl T., Marx D., Neumann R., Schroder S., Seelig P., Volker L. Precision laser spectroscopy of ground state hyperfine splitting of $\mathrm{H}$-like ${ }^{209} \mathrm{Bi}^{82+} / /$ Phys.Rev.Lett. - 1994. - Vol.73. - P.2425-2427.

5. Koshelev K.V., Labzowsky L.N., Tupitsyn I.I., The interelectron interaction corrections to the hyperfine structure of the 2p3/2 state in Li-like, B-like and N-like ГРАФОБЪЕКТions//J.Phys.B. - 2004. Vol.37. - P.843-851.

6. Ivanov L.N., Ivanova E.P., Kalinkin A.N. Nuclear and radiative effects in spectra of heavy and superheavy hydrogen-like ions//Nucl.Phys. - 1985. Vol.42. - P.355-362.

7. Ivanova E.P., Ivanov L.N., Glushkov A.V., Kramida A.E. High order corrections in the Relativistic Perturbation Theory with the model Zeroth Approximation, Mg-like and Ne-like ions //Phys.Scripta. 1985. - Vol.32(4). - P.512-524.

8. A.V.Glushkov, S.V.Ambrosov, A.V.Loboda A.V., Yu.G.Chernyakova, A.A.Svinarenko, O.Yu.Khetselius, QED calculation of the superheavy elements ions: energy levels, radiative corrections and hfs for different nuclear models// Nucl. Phys.A.: Nucl.and Hadr. Phys. - 2004. - Vol.734. - P.21-24.

9. Khetselius O.Yu., Hyper fine structure of radium// Photoelectronics. - 2005. - N14. - P.83-85.

10. Gurnitskaya E.P., Khetselius O.Y., Sensing the hyperfine structure and nuclear quadrupole moment for radium// Sensor Electr. and Microsyst. Techn. 2006. - N2. - P.25-29.

11. Glushkov A.V., Khetselius O., Consistent Quantum electrodynamics calculation of the fine and hyperfine structure parameters for heavy and super heavy multicharged Li-like ions// Preprint of I.I.Mechnikov
Odessa National University. Institute of Physics: PhL-4. - Odessa-2003.

12. Glushkov A.V., Ivanov L.N. Radiation Decay of Atomic States: atomic residue and gauge non-invariant contributions // Phys. Lett.A. - 1992. Vol.170(1). - P.33-38.

13. Glushkov A.V., Rusov V.D., Ambrosov S.V., Loboda A.V., Resonance states of compound super-heavy nucleus and EPPP in heavy nucleus collisions // New Projects and New Lines of research in Nuclear physics.Eds. Fazio G. and Hanappe F.,pp.142-154, Singapore, World Sci. (2003).

14. Glushkov A.V., Malinovskaya S.V., Svinarenko A.A., Chernyakova Yu.G., QED Calculation of Electron Satellites Spectra in Intense Laser Field in Multicharged Ion//Int.J.Quant.Chem. - 2004. Vol.99(4). - P.936-939.

15. Glushkov A.V., Malinovskaya S.V., Co-operative laser nuclear processes: border lines effects// In: New projects and new lines of research in nuclear physics. Eds. G.Fazio and F.Hanappe, pp.242-260, Singapore : World Sci. (2003).

16. Glushkov A.V. Negative Ions of inert Gases// Pis'ma to JETP. - 1992. - Vol.55. - P.104-107; JETP Lett. - 1992. - Vol.55(2). - P.97-100.

17. Sobel'man I.I. Introduction to theory of atomic spectra. - Moscow: Nauka. - 1977.

18. Glushkov A.V., Gurnitskaya E.P., Loboda A.V., Advanced quantum mechanical calculation of superheavy ions: energy levels, Radiation and Finite Nuclear size effects// Low Energy Antiproton Phys., AIP Serie. - 2005. - Vol.796. - P.217-220.

19. Glushkov A.V., Ambrosov S.V., Loboda A.V., Gurnitskaya E.P., Khetselius O.Yu., QED calculation of heavy multicharged ions with account for the correlation, radiative and nuclear effects// Recent Adv. in Theor. Phys. \&Chem. Systems. - 2006. - Vol.15. P.285-300.

20. Glushkov A.V., Malinovskaya S.V., Gurnitskaya E P., Khetselius O.Yu.,Dubrovskaya Yu.V., Consistent quantum theory of the recoil induced excitation and ionization in atoms during capture of neitron// J.Phys.CS. - 2006. - Vol.35. - P.425-430.

21. Glushkov A.V., Malinovskaya S.V., Dubrovskaya Yu.V., Vitavetskaya L.A., Quantum calculation of cooperative muon-nuclear processes: discharge of metastable nuclei during $\mu^{-}$capture// Recent Adv. in Theor. Phys.\&Chem. Systems. - 2006. - Vol.15. P.301-324.

22. Glushkov A.V., Ambrosov S.V., Loboda A.V., Gurnitskaya E.P., Prepelitsa G.P., Consistent QED approach to calculation of electron-collision excitation cross-sections and strengths: Ne-like ions // Int. Journ.Quant.Chem. - 2005. - Vol.104, N4 . - P. 562-569. 